\title{
MATHEMATICAL ANALYSIS OF EFFECTS OF DETECTION RATE ON DYNAMICS SPREAD OF HUMAN IMMUNODEFICIENCY VIRUS (HIV) AND ACQUIRE IMMUNODEFICIENCY SYNDROME (AIDS)
}

\author{
Adewale, Sunday O. \\ Department of Pure \&Applied Mathematics \\ LAUTECH Ogbomoso, Oyo State, Nigeria. \\ Omoloye, Musibau A. \\ Department of Statistics \\ Federal Polytechnic, Offa, Kwara State, Nigeria.
}

\begin{abstract}
Human Immuno-deficiency Virus (HIV) remains a major global health challenges, with an estimated incident rate of about $\mathbf{1 . 4}$ million patients worldwide. The progression of this infectious disease has resulted to increase in morbidity and mortality rate of the victims. The modeling the dynamics of HIV with sensitivity and detection of infected undetected individuals at a global population level. A new deterministic model was formulated which incorporated the undetected infected individuals to gain insight into the effect of detection rate of this class on the dynamical spread of HIV. Six compartmental of HIV models that comprises of susceptible, exposed, infected detected, infected undetected, treated and AIDS class was developed. Existence and uniqueness of solution to the model was examined quantitatively. The computation of sensitivity indices was carried out by normalized forward sensitivity index approach. Stability analysis was carried out for the disease free equilibrium and endemic equilibrium points. Basic reproduction number for the model was obtained by next generation matrix method (NGM). Numerical simulation was carried out by MAPLE 18.0 software using differential transformation method (DTM), to show the effect of epidemiological features and sensitive parameters on the dynamical spread of HIV infection.
\end{abstract}

Keywords-HIV, Equilibrium and Stability Analysis, Sensitivity, Basic Reproduction $R_{0}$.

\section{INTRODUCTION}

AIDS (acquired immune deficiency syndrome) is a disease caused by loss of immunity. It reduces immune system due to

\author{
Oladapo, Asimiyu O. \\ Department of Mathematical and Physical Sciences \\ Osun State University Osogbo, Osun State, Nigeria. \\ Ayoola, Tawakalit A. \\ Department of Mathematical and Physical Sciences \\ Osun State University, Osogbo, Osun State, Nigeria.
}

the HIV (Human Immunodeficiency Virus). HIV is an obligated parasite that can only live in cell or media. The virus comfortably live and multiplying inside a white blood cells. The virus used to be found inside body fluid that containing white blood cells such as vaginal fluid, sperm fluid, white blood cells, semen, breast fluid and cerebrospinal fluid. Furthermore the transmission occur when there is mixing of a body fluid that containing HIV. Especially, having sex with a partner that has HIV, contaminated needles and piercing tools with HIV, transfusion of blood and product that has HIV, and a pregnancy women that has HIV can transfer to a fuetus or baby inside placental. HIV has shown high degree of prevalence in population all over the world [6], since it was first identified in USA. Shortly after its detection AIDS became a global epidemic. About 90 percent of all HIV infected people live in the developing countries [8]. The total cases of AIDS related death recorded between 1981 to 2005 were estimated to about 25million, [7].

A recent global report gives an estimate of millions of people were living with HIV in 2012. An increase from previous years as more people are receiving the lifesaving antiretroviral therapy, there were 2.3 million new HIV infections globally showing a 33 percent decline in the number of new infections from 3.4 million in 2001. Also the number of AIDS death is also reducing from 2.3 million in 2005 to 1.6 million AIDS in 2012[9]. Sub-Saharan Africa has less than 11 percent of the world's population, containing 70 percent of the world's population of HIV-infected people. It remains the hardest hit region with 25.8 million HIV infected people with an estimate of 2.4 million death of HIV related illness, (Mukandavire \& Garira, 2007). The most populated HIV countries based on UNAID estimated in 2005, contribute 20 percent of Africa's 


\section{International Journal of Engineering Applied Sciences and Technology, 2020 \\ Vol. 5, Issue 4, ISSN No. 2455-2143, Pages 47-56 \\ Published Online August 2020 in IJEAST (http://www.ijeast.com)}

total AIDS infective, 10 percent of the world's HIV infective, with an estimate of about 3.86 million people living with HIV/AIDS, 221,000 AIDS related deaths and 370,000 new HIV infections annually, [2].

Up till now, there are no medicine or vaccination that can fight against the HIV/AIDS. What can be done for the people that are affected is to be maintain the condition of their body by eating nutritious foods, rest on time, recreation and exercising often.

\section{MODEL FORMULATIONS}

Considered six (6) compartmental deterministic HIV models that comprises of susceptible $\mathrm{S}(\mathrm{t})$, exposed $\mathrm{E}(\mathrm{t})$, infected detected $I_{D}(t)$, infected undetected $I_{U}(t)$, treated $T(t)$ and AIDS $\mathrm{A}(\mathrm{t})$ class.

Where

$N(t)=S(t)+E(t)+I_{D}(t)+I_{U}(t)+T(t)+A(t)$

Susceptible(S): Susceptible is a member of individual who is at risk of becoming infected by a disease. The population of susceptible of sexually active individuals at The recruitment of people (either by birth or immigration) into the population at rate $\pi$ and The susceptible population is increased while the population decrease by natural death rate $\mu$ and infected transmission which can be acquired following contact infectious only at rate $\lambda$

Latent(L): Latent individual is a member of a population who is infected but not infectious. The population of exposed individuals increases through the infection of susceptible and are assumed to show no disease symptoms initially fraction $\varepsilon_{1}$ . These individuals ("slow progressor") are moved to the latently HIV class. The newly infected individuals are assumed to immediately display disease symptoms (fast progressor) with the remaining fraction, $\left(1-\varepsilon_{1}\right)$ and are moved to the undetected infectious class. The population of latent class is decreased by the progression of latent HIV individual to active undetected HIV and also reduced by natural death rate and finally increased by the fraction of treated HIV that moves from treated class to latently HIV compartment.

Infected Undetected(IU $\left.\mathbf{I}_{\mathbf{U}}\right)$ : The undetected infected individuals of the population is increased by the infection of fast progressors at the rate $(1-\varepsilon) \lambda$ and the development of symptoms by individual latently. This population is decreased by natural death rate $(\mu)$, disease induced death rate $\delta$ and further decreased by detection rate $\gamma$ of HIV undetected infected individuals. Hence

Infected detected(I): The population of detected infected HIV individual by the fraction of latently individuals who develop the disease symptoms (at the rate $\omega_{1} v$ ) and detection of undetected individual at the rate $\gamma I_{U}$. The population later decreased by treatment rate $(\tau)$ for HIV detected individual and finally reduced by the natural death rate, induced mortality death rate at $\mu$ and $\delta$ respectively. Hence we have.

Treated class (T): The population of treated HIV individual is increased by those that have received treatment from HIV detected infected individual at the rate $(\tau)$ this population reduces by fraction of treated individual that moved back to latently HIV Individuals at the rate, $(\rho)$ since treatment does not completely clears the virus and finally reduced by natural and disease death rate $(\mu, \delta)$ respectively. Hence,

Aids (A): The full blown AIDS class is generated through the progression of individual from infected detected individuals that failed treatments at rate $\kappa$. It reduced by natural and disease death rate $(\mu, \delta)$ respectively. Hence,

Hence, we have the following nonlinear system of differential equations:

$$
\begin{aligned}
& \frac{d S}{d t}=\pi-\lambda S-\mu S \\
& \frac{d L}{d t}=(1-\varepsilon) \lambda S-(\mu+v) L+\rho T \\
& \frac{d \mathrm{I}_{U}}{d t}=\varepsilon \lambda S-(\mu+\delta+\gamma) I_{U}+\left(1-\omega_{1}\right) v L \\
& \frac{d \mathrm{I}_{D}}{d t}=\omega_{1} v L-(\mu+\delta+\tau+\kappa) I_{D}+\gamma I_{U} \\
& \frac{d T}{d t}=\tau \mathrm{I}_{D}-(\mu+\delta+\rho) \mathrm{T} \\
& \frac{d \mathrm{~A}}{d t}=\kappa_{D}-(\delta+\mu) \mathrm{A}
\end{aligned}
$$

Where force of infection is given as:

$$
\lambda=\frac{\beta\left(L+\eta_{U} I_{U}+\eta_{D} I_{D}+\eta_{T} T+\eta_{A} A\right)}{N}
$$

In equation (3), $\beta$ represents the effective contact rate i. e contact capable of leading to HIV infection

Table 1. Description of Parameter

\begin{tabular}{ll}
\hline Parameters & Definition \\
\hline$\pi$ & Recruitment rate \\
$\mu$ & Natural death rate \\
$\beta$ & Effective contact rate \\
$\omega_{1}$ & $\begin{array}{l}\text { Progression rate from infected } \\
v\end{array}$ \\
& undetected to infected detected \\
$\varepsilon$ & undetected rogresion rate from latent to infected \\
\hline
\end{tabular}




\begin{tabular}{ll}
\hline$\tau$ & Treatment rate \\
$\kappa$ & $\begin{array}{l}\text { progression rate from infected detected } \\
\text { stage to AIDS class }\end{array}$ \\
$\delta$ & Disease induced death rate \\
$\gamma$ & Detection rate \\
$\eta_{U}, \eta_{D}, \eta_{T}, \eta_{A}$ & Modification parameters \\
$\rho$ & Loss of immunity of treated individual \\
\hline
\end{tabular}

Table 2. Description of Variables

\begin{tabular}{ll}
\hline VARIABLE & DEFINITION \\
\hline$S$ & Susceptible \\
$L$ & Latently infected Population \\
$I_{U}$ & Infected undetected Population \\
$I_{D}$ & Infected detected Population \\
$T$ & Treated Population \\
$A$ & AIDS Population \\
\hline
\end{tabular}

III. MODEL ANALYSIS

\section{Positivity invariant solution}

Theorem1: The closed set $\quad$ D
$\left\{\left(\boldsymbol{S}, \boldsymbol{L}, \boldsymbol{I}_{\boldsymbol{U}}, \boldsymbol{I}_{\boldsymbol{D}}, \boldsymbol{T}, \boldsymbol{A}\right) \in \mathfrak{R}_{+}^{\sigma}: N \leq \frac{\pi}{\mu}\right\}$ is

positive invariant and attracting with respect to the model (2)

Proof: Adding all the equation in the model (2) gives

$\frac{d \mathrm{~N}}{d t}=\frac{d S}{d t}+\frac{d L}{d t}+\frac{d I_{U}}{d t}+\frac{d I_{D}}{d t}+\frac{d T}{d t}+\frac{d A}{d t}$

$N=S+L+I_{U}+I_{D}+T+A=-\mu$

$\frac{d N}{d t}=\pi-\mu \mathrm{N}-\gamma\left(I_{U}+I_{D}+\mathrm{T}+\mathrm{A}\right)-\tau\left(I_{D}-I\right)$

In the absence of HIV infection, equation (5) implies:

$\frac{d N}{d t}=\pi-\mu N$

$\frac{d N}{d t}+\mu \mathrm{N}=\pi$

I.F $=\ell^{\int \rho(t) d t}=\ell^{\mu t}$

Multiplying both sides of equation with $\ell^{\mu t}$

$\ell^{\mu t} \frac{d N}{d t}+\mu N \ell^{\mu t}=\pi \ell^{\mu t}$

Now integrating both sides

$\mathrm{N}(\boldsymbol{t}) \ell \mu t=\frac{\pi}{\mu} \ell^{\mu t}+C$

Therefore, $N(t)=\frac{\pi}{\mu}+c \ell^{\mu t}$

Using the initial condition, that when $\mathrm{t}=0, \mathrm{~N}(\mathrm{t})=\mathrm{N}(0)$ to obtain $N(\mathrm{O}) \leq \frac{\pi}{\mu}+c$ i.e $C=N(0) \frac{\pi}{\mu}$

i.e $N \leq \frac{\pi}{\mu}+\left(N(0)-\frac{\pi}{\mu}\right) \ell^{\mu t}$

Equation (7) becomes

$N \leq \frac{\pi}{\mu}+\left(N(0)-\frac{\pi}{\mu}\right) \ell^{-\mu t}$

Applying Kirchhoff and Pota's theorem on the above inequality at $t \rightarrow \infty$, to obtain $N \leq \frac{\pi}{\mu}$. Hence all the feasible solutions of the model (2) enter the region $D=\left\{\left(S, L, I_{U}, I_{D}, T, A\right): N \leq \frac{\pi}{\mu}\right\}$, which is positively invariant, attracting and it's sufficient to consider solutions in $D$ (Hethcote, 2000).

Furthermore, existence, uniqueness and continuation of result for model (2) hold in this region D (Maliyoni et al, 2012). Thus the model (2) is mathematically, epidemiology meaningful and the dynamics of the model (2) can now be studied in the domain D.

\section{Existence of Disease Free Equilibrium (DFE)}

Let $\varepsilon_{0}$ represent the equilibrium point at DFE in the absence of infection, $L=I_{U}=I_{d}=T=A=0$ and $S=\frac{\pi}{\mu}$ from (2). The model (2) has its DFE given by

$$
\varepsilon_{0}=\left(S, L, I_{U}, I_{D}, T, A\right)=\left(\frac{\pi}{\mu}, 0,0,0,0,0\right)
$$

Existence of Endemic Equilibrium (EPP)

In this section, the possible existence and stability of endemic (positive) equilibrium of model equation equilibria where at least one of the infected components of the model is non-zero will be considered.

Let $\quad \varepsilon_{1}^{* *}=\left(S^{* *}, L^{* *}, I_{U}^{* *}, I_{d}^{* *}, T^{* *}, A^{* *}\right)$ represents any arbitrary endemic equilibrium of the model equation solving the equations of the system at steady state thus; 
$\frac{d s}{d t}=\pi-S(\lambda+\mu) \Rightarrow S^{* *}=\frac{\pi}{\lambda+\mu}$

$\frac{d L}{d t}=(1-\varepsilon) \lambda S-(\mu+v) L+\rho T \Rightarrow L^{* *}=\frac{(1-\varepsilon) \lambda S}{C_{2}}$

$\frac{d I_{U}}{d t}=\varepsilon \lambda S-I_{U}(\mu+\delta+\gamma)+\left(1-\omega_{1}\right) \nu L \Rightarrow I_{U}=\frac{\varepsilon \lambda S+\left(1-\omega_{1}\right) \nu L}{C_{3}}$

$\frac{d I_{d}}{d t}=\omega_{1} \nu L-I_{D}(\mu+\delta-\gamma+\tau)-\kappa I \Rightarrow I_{D}=\frac{\omega_{1} \nu L-\kappa I}{C_{4}}$

$\frac{d T}{d t}=\tau I-T(\mu+\delta+\rho) \Rightarrow T=\frac{\tau I}{C_{5}}$

$\frac{d A}{d t}=\kappa l-A(\delta+\mu) \Rightarrow A=\frac{\kappa l}{C_{6}}$

the expression for $\lambda$ at the endemic steady-state, denoted by $\lambda^{* *}$ is given by

$\lambda^{* * *}=\frac{\beta\left(L^{* * *}+\eta_{1} I_{U}^{* * *}+\eta_{2} I_{D}^{* * *}+\eta_{3} T^{* * *}+\eta_{4} A^{* * *}\right)}{N}$

$N^{* * *}=S^{* * *}+L^{* * *}+I_{U}^{* * *}+I_{D}^{* * *}+T^{* * *}+A^{* * *}$

For computational convenience, we re-write expression $* *$ in terms of $\lambda^{* * *} S^{* * *}$ as below:

$L^{* * *}=\frac{(1-\varepsilon) \lambda^{* * *} S^{* *}}{C_{2}}=P_{1} \lambda^{* * *} S^{* * *}$

$I_{U}^{* *}=\frac{\varepsilon \lambda^{* *} S^{* *}}{C_{3}}+\frac{v\left(1-\omega_{1}\right)(1-\varepsilon) \lambda^{* * *} S^{* *}}{C_{2} C_{3}}=P_{2} \lambda^{* *} S^{* *}$

$I_{D}^{* * *}=\frac{\omega_{1} v(1-\varepsilon) \lambda^{* * *} S^{* * *}}{C_{2} C_{4}}-\frac{\kappa P_{2} \lambda^{* * *} S^{* * *}}{C_{4}}=P_{3} \lambda^{* *} S^{* * *}$

$T^{* *}=\frac{\tau P_{3} \lambda^{* *} S^{* *}}{C_{5}}=P_{4} \lambda^{* *} S^{* * *}$

Where;

$P_{1}=\frac{(1-\varepsilon)}{C_{2}} \quad P_{2}=\frac{v\left(1-\omega_{1}\right)(1-\varepsilon)}{C_{2} C_{3}} \quad P_{3}=\frac{\omega_{1} v(1-\varepsilon)}{C_{2} C_{4}}-\frac{\kappa P_{2}}{C_{4}} P_{4}=\frac{\tau P_{3}}{C_{5}}$

$C_{1}=\lambda+\mu, C_{2}=\mu+v, C_{3}=(\mu+\delta+\gamma), C_{4}=(\mu+\delta-\gamma+\tau), C_{5}=(\mu+\delta+\rho), C_{6}=(\delta+\mu)$

Substituting the expressions in (10) into (9) gives

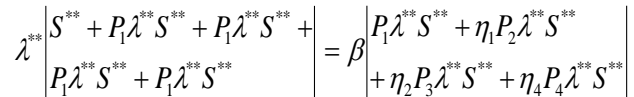

Substracting from both sides and divide both sides by $\lambda^{* * * *} S^{* * *}$ obtain

$1+P_{2}+P_{3}+P_{4}=\beta\left|\eta_{1} P_{2}+\eta_{2} P_{3}+\eta_{4} P_{4}\right|$

Let $\quad P_{2}+P_{3}+P_{4}=P_{5}$

Therefore;

$1+P_{5} \lambda^{* *}=\beta\left|\eta_{1} P_{2}+\eta_{2} P_{3}+\eta_{4} P_{4}\right|=R_{0}$

So that $\lambda^{* * *}=\frac{R_{0}-1}{P_{5}}>0$

Whenever $R_{0}>1$
The component of the unique endemic equilibrium $\left(\varepsilon_{1}\right)$ can be obtained by substituting the unique value of $\lambda^{* *}$ into the expressions in $\left(S^{* *}, L^{* *}, I_{U}^{* *}, I_{D}^{* *}, T^{* *}\right)$. Thus the following result has been established.

\section{Local Stability of Disease Free Equilibrium.}

The stability of disease free equilibrium was done by linearization; the characteristics root of the polynomial was examined.

$$
\begin{aligned}
& \frac{d S}{d t}=\pi-\mu S \\
& \frac{d L}{d t}=-(\mu+v) L+\rho T \\
& \frac{d \mathrm{I}_{U}}{d t}=-(\mu+\delta+\gamma) I_{U}+\left(1-\omega_{1}\right) v L \\
& \frac{d \mathrm{I}_{D}}{d t}=\omega_{1} v L-(\mu+\delta+\tau+\kappa) I_{D}+\gamma I_{U} \\
& \frac{d T}{d t}=\tau \mathrm{I}_{D}-(\mu+\delta+\rho) \mathrm{T}
\end{aligned}
$$

$\left(\begin{array}{ccccc}-\mu & 0 & 0 & 0 & 0 \\ 0 & -(\mu+v) & 0 & 0 & 0 \\ 0 & -\omega v & -(\mu+\delta+\tau) & 0 & 0 \\ 0 & 0 & -\gamma & -(\mu+\delta+\rho) & 0 \\ 0 & 0 & 0 & 0 & -(\delta+\mu)\end{array}\right)$

the characteristics equation is determined by $|J-I \lambda|$ where is I $5 \times 5$ identity matrix.

$$
\left|\begin{array}{ccccc}
-\mu-\lambda & 0 & 0 & 0 & 0 \\
0 & -(\mu+v)-\lambda & 0 & 0 & 0 \\
0 & -\omega v & -(\mu+\delta+\tau)-\lambda & 0 & 0 \\
0 & 0 & -\gamma & -(\mu+\delta+\rho)-\lambda & 0 \\
0 & 0 & 0 & 0 & -(\delta+\mu)-\lambda
\end{array}\right|=0
$$

$-\mu-\lambda\left|\begin{array}{cccc}-(\mu+v)-\lambda & 0 & 0 & 0 \\ -\omega v & -(\mu+\delta+\tau)-\lambda & 0 & 0 \\ 0 & -\gamma & -(\mu+\delta+\rho)-\lambda & 0 \\ 0 & 0 & 0 & -(\delta+\mu)-\lambda\end{array}\right|=0$

$$
-(\mu-\lambda)[-(v+\mu)-\lambda]\left|\begin{array}{ccc}
-(\mu+\delta+\tau)-\lambda & 0 & 0 \\
-\gamma & -(\mu+\delta+\rho)-\lambda & 0 \\
0 & 0 & -(\delta+\mu)-\lambda
\end{array}\right|=0
$$

$-(\mu-\lambda)[-(v+\mu)-\lambda][-(\mu+\delta+\tau)-\lambda]\left|\begin{array}{cc}-(\mu+\delta+\rho)-\lambda & 0 \\ 0 & -(\delta+\mu)-\lambda\end{array}\right|=0$ 
$[-(\mu-\lambda)][-(\delta+\mu)-\lambda][-(\mu+\delta+\tau)-\lambda][-(\mu+\delta+\rho)-\lambda][-(\delta+\mu)-\lambda]=0$

$\lambda_{1}=-\mu$

$\lambda_{2}=-(v+\mu)$

$\lambda_{3}=-(\mu+\delta+\tau)$

$\lambda_{4}=-(\mu+\delta+\rho)$

$\lambda_{5}=-(\delta+\mu)$

Since $\lambda_{1}, \lambda_{2}, \lambda_{3}, \lambda_{4}$, and $\lambda_{5}$ are negative real and distinct, it shows that the model is stable if $R_{0}<1$, then the diseases free equilibrium $\varepsilon_{0}=\left(S_{0}, 0,0,0,0,0\right)$ of the model is locally asymptotically stable, but if $R_{0}>1, \varepsilon_{0}$ is unstable and there exist an endemic equilibrium $\varepsilon_{1}^{* *}=\left(S^{* * *}, L^{* * *}, I_{U}^{* * *}, I_{d}^{* * *}, T^{* * *}\right)$

Global Stability Analysis of the Disease Free Equilibrium Here, the global stability (GAS) property of DEF of the HIV model only will be explored.

Theorem 2: The disease free equilibrium of the system (2) is globally asymptotically stable whenever $R_{0}<1$ and unstable $R_{0}>1$.

Proof: it follows that $S=N-L-I_{U}-I_{D}-T-A$ at the steady state. The proof is based on using the comparison theorem (Lakshmkantham et al, 1999) to prove the global stability. The rate of change of the variables representing the infected component of the system can be written as follows.

$\frac{d L}{d t}=(1-\varepsilon) \lambda S-(\mu+v) L+\rho T$

$\frac{d \mathrm{I}_{U}}{d t}=\varepsilon \lambda S-(\mu+\delta+\gamma) I_{U}+\left(1-\omega_{1}\right) v L$

$\frac{d \mathrm{I}_{D}}{d t}=\omega_{1} v L-(\mu+\delta+\tau+\kappa) I_{D}+\gamma I_{U}$

$\frac{d T}{d t}=\tau \mathrm{I}_{D}-(\mu+\delta+\rho) \mathrm{T}$

$\frac{d \mathrm{~A}}{d t}=\kappa \mathrm{d}_{D}-(\delta+\mu) \mathrm{A}$

$\left.D^{*}=\left\{L, I_{U}, I_{D}, T, A\right) \in \mathfrak{R}_{+}^{5}: L+I_{U}+I_{D}+T \leq N^{*}\right\}$

For the model (3.8), the associated reproduction number, denoted by $R_{0}$ where

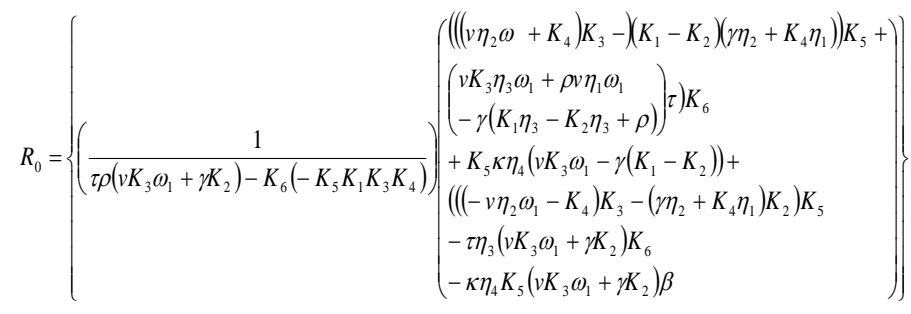

The DFE of the model (2) given by disease free, is GAS in $\mathrm{D}^{*}$ if $R_{0}<1$

Using comparison method, obtain

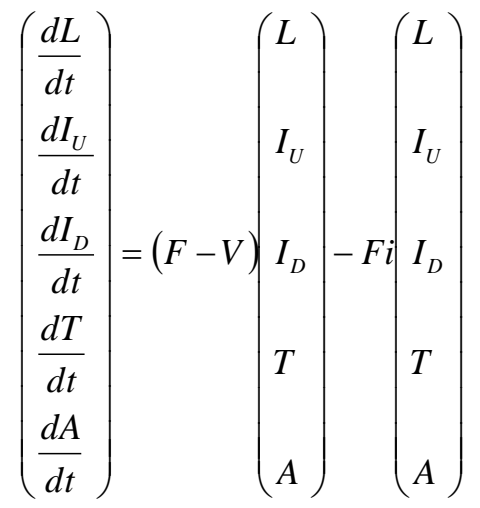

Where

$F=\left(\begin{array}{ccccc}(1-\varepsilon) \beta & (1-\varepsilon) \beta \eta_{1} & (1-\varepsilon) \varepsilon \eta_{2} & (1-\varepsilon) \beta \eta_{3} & (1-\varepsilon) \beta \eta_{4} \\ \varepsilon \beta & \varepsilon \beta \eta_{1} & \varepsilon \beta \eta_{2} & \varepsilon \beta \eta_{3} & \varepsilon \beta \eta_{4} \\ 0 & 0 & 0 & 0 & 0 \\ 0 & 0 & 0 & 0 & 0 \\ 0 & 0 & 0 & 0 & 0\end{array}\right)$

$V=\left(\begin{array}{ccccc}K_{1} & 0 & 0 & -\rho & 0 \\ K_{2} & K_{3} & 0 & 0 & 0 \\ -\omega_{1} v & -\gamma & K_{4} & 0 & 0 \\ 0 & 0 & -\tau & K_{5} & 0 \\ 0 & 0 & -\kappa & 0 & K_{6}\end{array}\right)$

$(F-V)=\left(\begin{array}{ccccc}(1-\varepsilon) \beta-K_{1} & (1-\varepsilon) \beta \eta_{1} & (1-\varepsilon) \varepsilon \eta_{2} & (1-\varepsilon) \beta \eta_{3} & (1-\varepsilon) \beta \eta_{4} \\ \varepsilon \beta+K_{2} & \varepsilon \beta \eta_{1}-K_{3} & \varepsilon \beta \eta_{2} & \varepsilon \beta \eta_{3} & \varepsilon \beta \eta_{4} \\ \omega_{1} v & 0 & -K_{4} & 0 & 0 \\ 0 & 0 & \tau & -K_{5} & 0 \\ 0 & 0 & \kappa & 0 & -K_{6}\end{array}\right)$

Then, obtain 


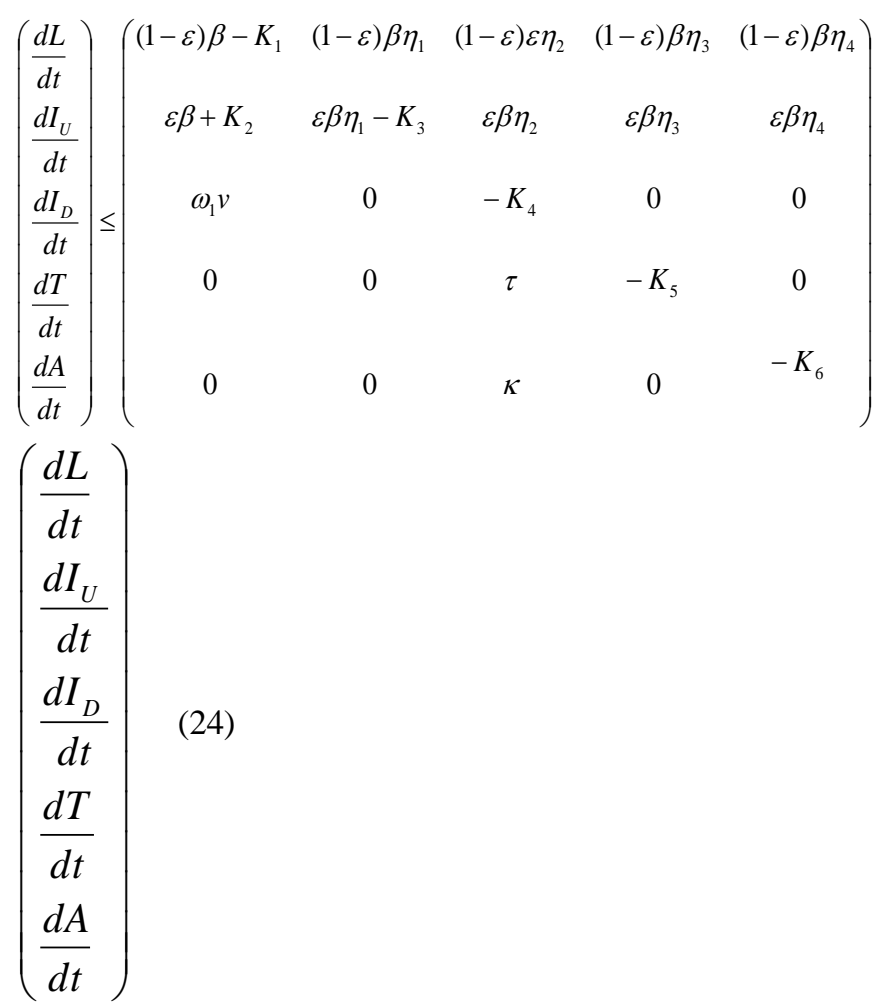

According to (Castillo Chavez et al 2002) and (Driessche \& Watmough 2002), all eigen values of the matrix (F-V) have negatives real parts.

$\left(\begin{array}{ccccc}\left((1-\varepsilon) \beta-K_{1}\right)-\lambda & (1-\varepsilon) \beta \eta_{1} & (1-\varepsilon) \varepsilon \eta_{2} & (1-\varepsilon) \beta \eta_{3} & (1-\varepsilon) \beta \eta_{4} \\ \varepsilon \beta+K_{2} & \left(\varepsilon \beta \eta_{1}-K_{3}\right)-\lambda & \varepsilon \beta \eta_{2} & \varepsilon \beta \eta_{3} & \varepsilon \beta \eta_{4} \\ \omega_{1} v & 0 & -K_{4}-\lambda & 0 & 0 \\ 0 & 0 & \tau & -K_{5}-\lambda & 0 \\ 0 & 0 & \kappa & 0 & -K_{6}-\lambda\end{array}\right)=0$

From equation (25), lead to

$\left[\begin{array}{l}\left.\left.\left((1-\varepsilon) \beta-K_{1}\right)-\lambda\right)\left(\varepsilon \beta \eta_{1}-K_{3}\right)-\lambda\right)\left(-K_{4}-\lambda\right)\left(-K_{5}-\lambda\right)\left(-K_{6}-\lambda\right)-(1-\varepsilon) \beta \eta_{1} \\ \left.\left(\varepsilon \beta+K_{2}\right)\left(-K_{4}-\lambda\right)\left(-K_{5}-\lambda\right)\left(-K_{6}-\lambda\right)-(1-\varepsilon) \varepsilon \eta_{2}\left(\varepsilon \beta \eta_{1}-K_{3}\right)-\lambda\right)\left(-K_{5}-\lambda\right) \\ \left(-K_{6}-\lambda\right)-(1-\varepsilon) \beta \eta_{3}\left(-K_{4}-\lambda\right)\left(-K_{6}-\lambda\right)-(1-\varepsilon) \beta \eta_{4}\left(\left(\varepsilon \beta \eta_{1}-K_{3}\right)-\lambda\right. \\ \left(-K_{4}-\lambda\right)\left(-K_{5}-\lambda\right)\end{array}\right]=0$

The equation (26), the characteristic equation is given by

$\left(\begin{array}{l}\left(\left(\left(v \eta_{2} \omega+K_{4}\right) K_{3}-\right)\left(K_{1}-K_{2}\right)\left(\gamma \eta_{2}+K_{4} \eta_{1}\right)\right) K_{5}+ \\ \left.\left(v K_{3} \eta_{3} \omega_{1}+\rho v \eta_{1} \omega_{1}-\gamma\left(K_{1} \eta_{3}-K_{2} \eta_{3}+\rho\right)\right) \tau\right) K_{6} \\ +K_{5} \kappa \eta_{4}\left(v K_{3} \omega_{1}-\gamma\left(K_{1}-K_{2}\right)\right)+\left(\left(\left(-v \eta_{2} \omega_{1}-K_{4}\right) K_{3}-\left(\gamma \eta_{2}+K_{4} \eta_{1}\right) K_{2}\right) K_{5}\right. \\ \left.-\tau \eta_{3}\left(v K_{3} \omega_{1}+\gamma K_{2}\right)\right) K_{6}-\kappa \eta_{4} K_{5}\left(v K_{3} \omega_{1}+\gamma K_{2}\right) \beta\end{array}\right)=0$

Let $\lambda^{5}+a_{5} \lambda^{4}+a_{4} \lambda^{3}+a_{3} \lambda^{2}+a_{2} \lambda+a_{1}$

Applying Routh-Hurtwitz criteria of order 5;

Where

$n=a_{1}>0, a_{3}>0, a_{4}>0, a_{5}>0$, and $a_{1} a_{2} a_{3} a_{4}>a_{3}^{2}+a_{1}^{2} a_{5}$.

Thus according to Routh Hurwitz Criteria of order five (5),

Then for $a_{1}>0$;

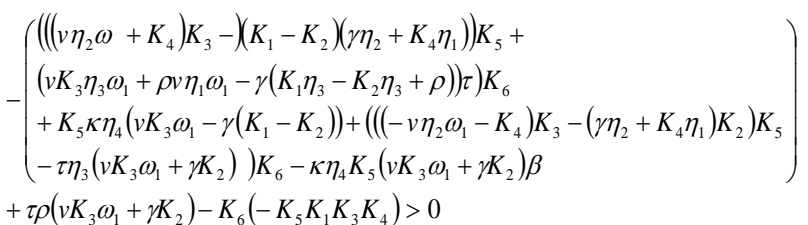

This lead to

$\left(\left(\left(v \eta_{2} \omega+K_{4}\right) K_{3}-\right)\left(K_{1}-K_{2}\right)\left(\gamma \eta_{2}+K_{4} \eta_{1}\right)\right) K_{5}+$

$\left.\left(v K_{3} \eta_{3} \omega_{1}+\rho v \eta_{1} \omega_{1}-\gamma\left(K_{1} \eta_{3}-K_{2} \eta_{3}+\rho\right)\right) \tau\right) K_{6}$

$+K_{5} \kappa \eta_{4}\left(v K_{3} \omega_{1}-\gamma\left(K_{1}-K_{2}\right)\right)+\left(\left(\left(-v \eta_{2} \omega_{1}-K_{4}\right) K_{3}-\left(\gamma \eta_{2}+K_{4} \eta_{1}\right) K_{2}\right) K_{5}>>\right.$

$\left.-\tau \eta_{3}\left(v K_{3} \omega_{1}+\gamma K_{2}\right)\right) K_{6}-\kappa \eta_{4} K_{5}\left(v K_{3} \omega_{1}+\gamma K_{2}\right) \beta$

$-\tau \rho\left(v K_{3} \omega_{1}+\gamma K_{2}\right)-K_{6}\left(-K_{5} K_{1} K_{3} K_{4}\right)$

Hence

$\frac{-\left(\begin{array}{l}\left.\left(\left(v \eta_{2} \omega+K_{4}\right) K_{3}-\right)\left(K_{1}-K_{2}\right)\left(\gamma \eta_{2}+K_{4} \eta_{1}\right)\right) K_{5}+ \\ \left.\left(v K_{3} \eta_{3} \omega_{1}+\rho v \eta_{1} \omega_{1}-\gamma\left(K_{1} \eta_{3}-K_{2} \eta_{3}+\rho\right)\right) \tau\right) K_{6} \\ +K_{5} \kappa \eta_{4}\left(v K_{3} \omega_{1}-\gamma\left(K_{1}-K_{2}\right)\right)+\left(\left(\left(-v \eta_{2} \omega_{1}-K_{4}\right) K_{3}-\left(\gamma \eta_{2}+K_{4} \eta_{1}\right) K_{2}\right) K_{5}\right. \\ \left.-\tau \eta_{3}\left(v K_{3} \omega_{1}+\gamma K_{2}\right)\right) K_{6}-\kappa \eta_{4} K_{5}\left(v K_{3} \omega_{1}+\gamma K_{2}\right) \beta\end{array}\right)}{\left(\tau \rho\left(v K_{3} \omega_{1}+\gamma K_{2}\right)-K_{6}\left(-K_{5} K_{1} K_{3} K_{4}\right)\right)}<1$,

Implying that $R_{0}<1$

According to [1] and (Driessche and Watmough 2002), all eigenvalues of the matrix $\mathrm{F}-\mathrm{V}$ have negative real parts. It follows that the linearized differential inequality above is stable whenever $R_{0}<1$.Consequently

$S=\left(L=I_{U}=I_{D}=T=A=0\right) \rightarrow(0,0,0,0,0)$

$t \rightarrow \infty$. Substituting $L=I_{U}=I_{D}=T=A=0$

In $\left(R_{0}\right)$ gives $S(t) \rightarrow S(0)$ as $t \rightarrow \infty$. Hence, the disease free equilibrium is globally asymptotically stable $R_{\mathrm{O}}<\mathbf{1}$ and unstable $\boldsymbol{R}_{\mathrm{o}}>\mathbf{1}$.

\section{Sensitivity Analysis}

It is used to conduct an investigation to determine how sensitive the threshold quantity basic reproduction number is with respect to its parameters, this will help us to know which of the parameters causes most reduction in $R_{0}$ parameters that have high impact on $\boldsymbol{R}_{\mathrm{O}}$ and these should be targeted by intervention strategies in order to have most effective control of the disease. This analysis tells us how crucial and important each parameter is to disease transmission. The normalized forward sensitivity index of the reproduction number with respect to its parameters was computed which defined as:

$X_{W}^{R_{0}}=\frac{\partial R_{0}}{\partial w} \times \frac{w}{R_{0}}$

Sensitivity values for each parameter involved in $R_{0}$ is calculated and result were presented in the table below. 
Table 3. Numerical values of sensitivity analysis for HIV Parameter Sensitivity value

\begin{tabular}{lc}
\hline$\beta$ & 1.000000 \\
$\delta$ & -0.0078965 \\
$\omega$ & 0.090673 \\
$\tau$ & -0.141060 \\
$\kappa$ & -0.699705 \\
$\gamma$ & -0.0838820 \\
$\varepsilon$ & 0.763369 \\
$\eta_{U}$ & 0.0000003 \\
$\eta_{D}$ & 0.000012 \\
$\eta_{T}$ & 0.000410 \\
\hline
\end{tabular}

NUMERICAL SIMULATION

In this research, the six (6) nonlinear differential equations were obtained using some of the parameter values in table 4 . The simulation was carried out using the Maple 18 software in order to determine the dynamical spread pattern of the disease in the population and to know which of the disease parameters should be given attention by the public and policy health makers.

Table 4. Numerical values of Parameter

\begin{tabular}{ll} 
Parameter & Value \\
\hline$\pi$ & 3000 \\
$\mu$ & 0.2 \\
$\beta$ & 0.333 \\
$\delta$ & 0.2 \\
$\omega$ & 0.1 \\
$\tau$ & 0.2522 \\
$\kappa$ & 0.5 \\
$\gamma$ & 0.7 \\
$\varepsilon$ & 0.2 \\
$\nu$ & 0.1 \\
\hline
\end{tabular}

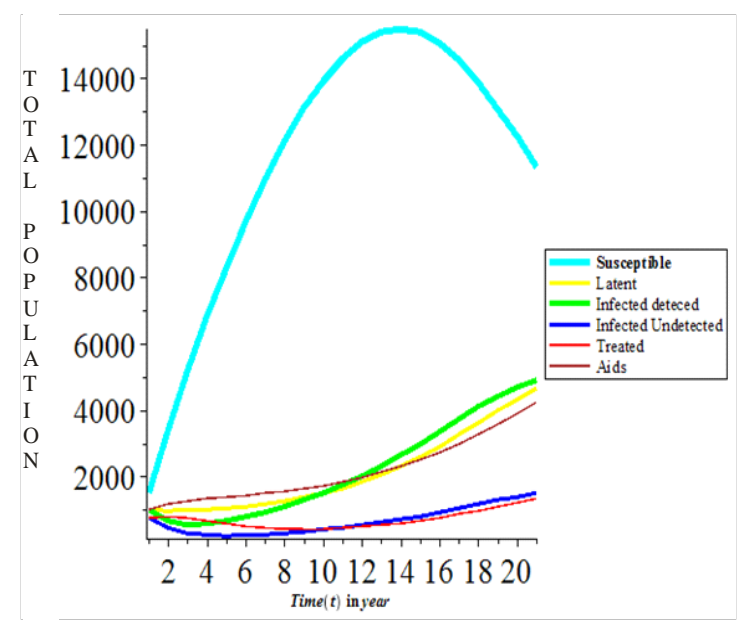

Figure 1: Plots of Total Population against time $\mathbf{t}$ for various value of $\beta(\mathbf{0 . 3 )}$

$\varepsilon=0.7, \eta_{U}=0.001, \eta_{D}=0.001, \eta_{T}=0.001, \tau=0.30619, \omega=0.2$, $\kappa=0.2522, \pi=2000, \delta=0.3, \gamma=0.2, \rho=0.12, v=0.12, \mu=0.02$

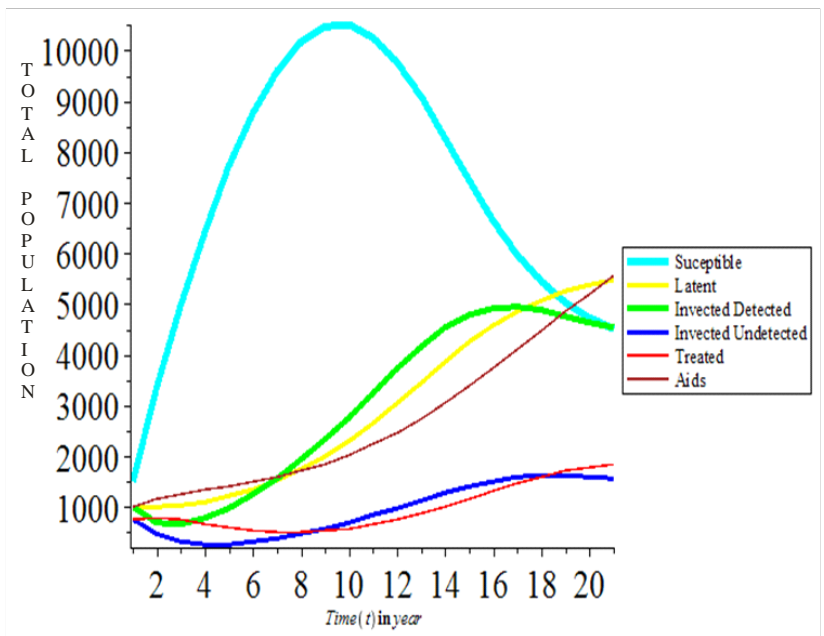

Figure 2: Plots of Total Population against time $\mathbf{t}$ for various value of $\beta(0.5)$

$\varepsilon=0.7, \eta_{U}=0.001, \eta_{D}=0.001, \eta_{T}=0.001, \tau=0.30619, \omega=0.2$, $\kappa=0.2522, \pi=2000, \delta=0.3, \gamma=0.2, \rho=0.12, v=0.12, \mu=0.02$ 
Published Online August 2020 in IJEAST (http://www.ijeast.com)

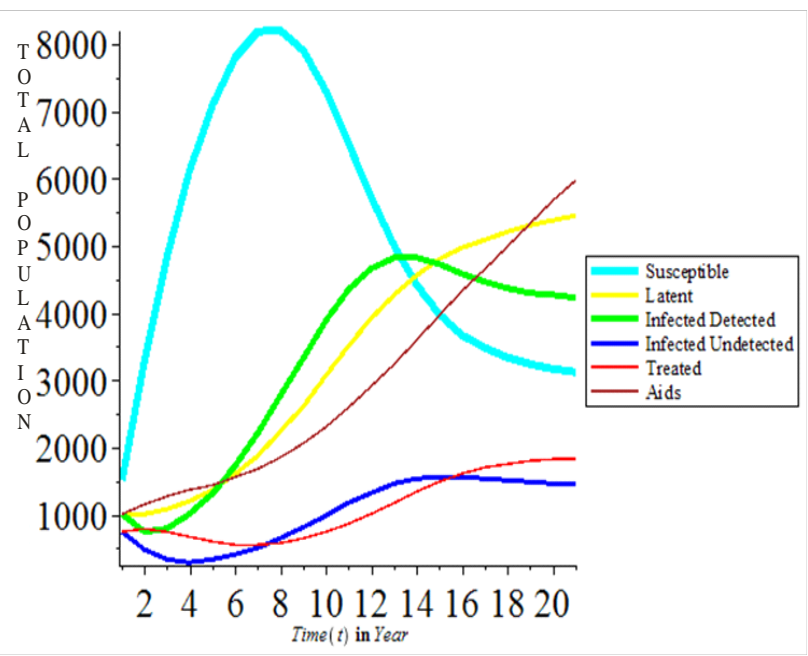

Figure 3: Plots of Total Population against time $t$ for various value of $\beta(0.7)$

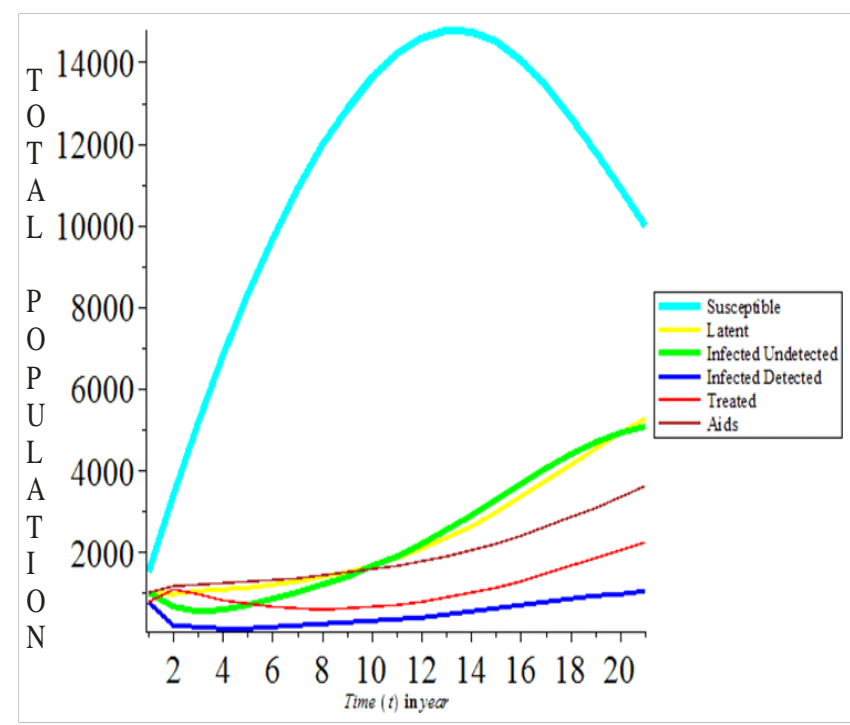

Figure 5: Plots of Total Population against time $t$ for various $\varepsilon=0.7, \eta_{U}=0.001, \eta_{D}=0.001, \eta_{T}=0.001, \tau=0.30619, \omega=0.2$, value of $\tau(0.70619)$ $\kappa=0.2522, \pi=2000, \delta=0.3, \gamma=0.2, \rho=0.12, v=0.12, \mu=0.02 \varepsilon=0.7, \beta=0.3, \eta_{U}=0.001, \eta_{D}=0.001, \eta_{T}=0.001, \omega=0.2$,

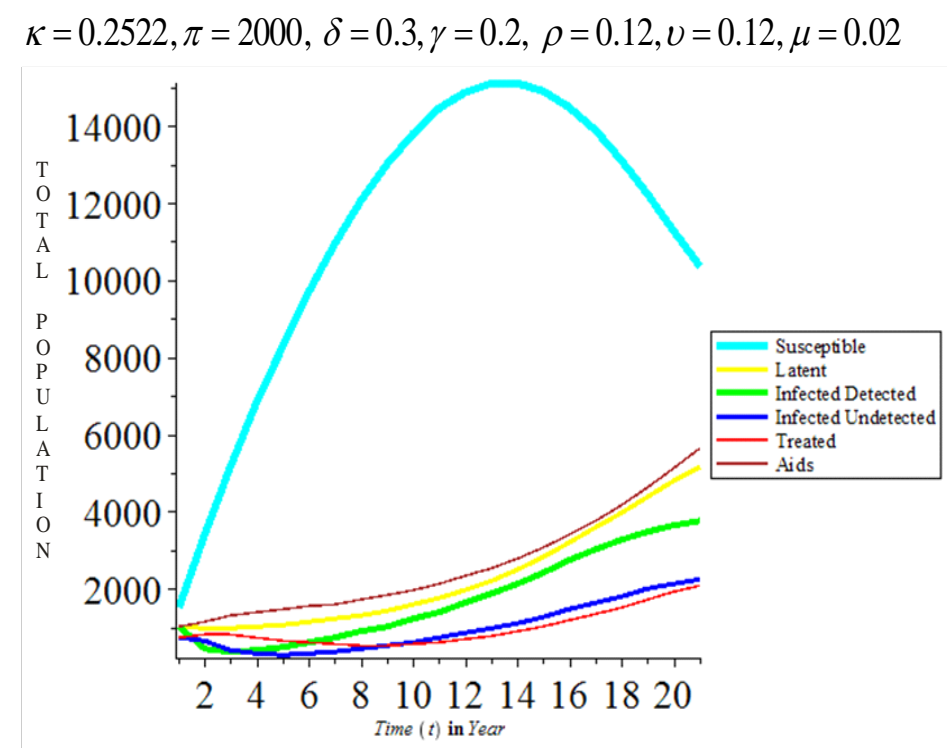

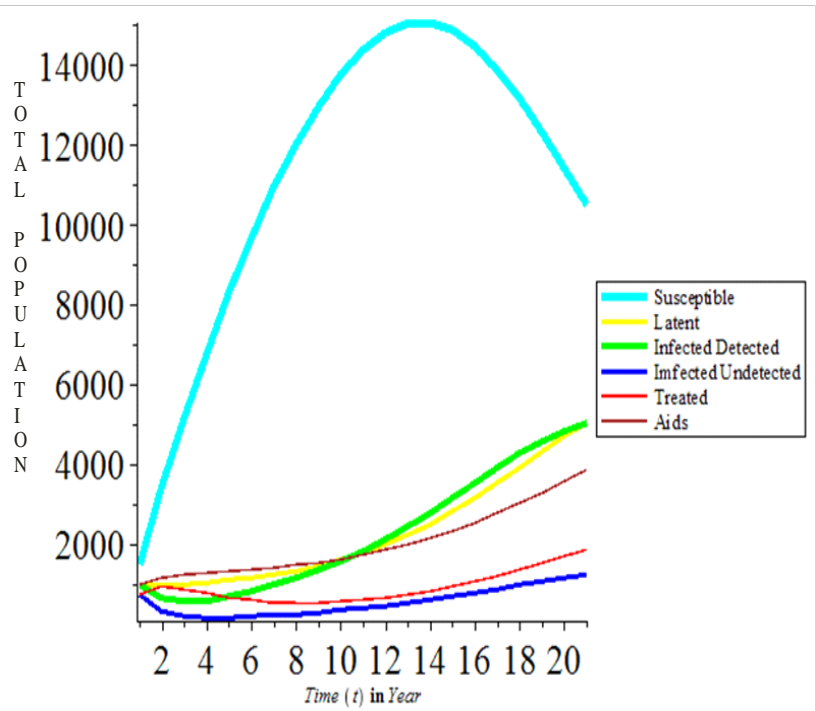

Figure 4: Plots of Total Population against time t for various value of $\tau(0.30619)$

$\varepsilon=0.7, \beta=0.3, \eta_{U}=0.001, \eta_{D}=0.001, \eta_{T}=0.001, \omega=0.2$,

$\kappa=0.2522, \pi=2000, \delta=0.3, \gamma=0.2, \rho=0.12, v=0.12, \mu=0.02$
Figure 6: Plots of Total Population against time $t$ for various value of $\gamma_{(0.4)}$ $\varepsilon=0.7, \eta_{U}=0.001, \eta_{D}=0.001, \eta_{T}=0.001, \tau=0.30619, \omega=0.2$, $\kappa=0.2522, \pi=2000, \delta=0.3, \rho=0.12, v=0.12, \mu=0.02$ 


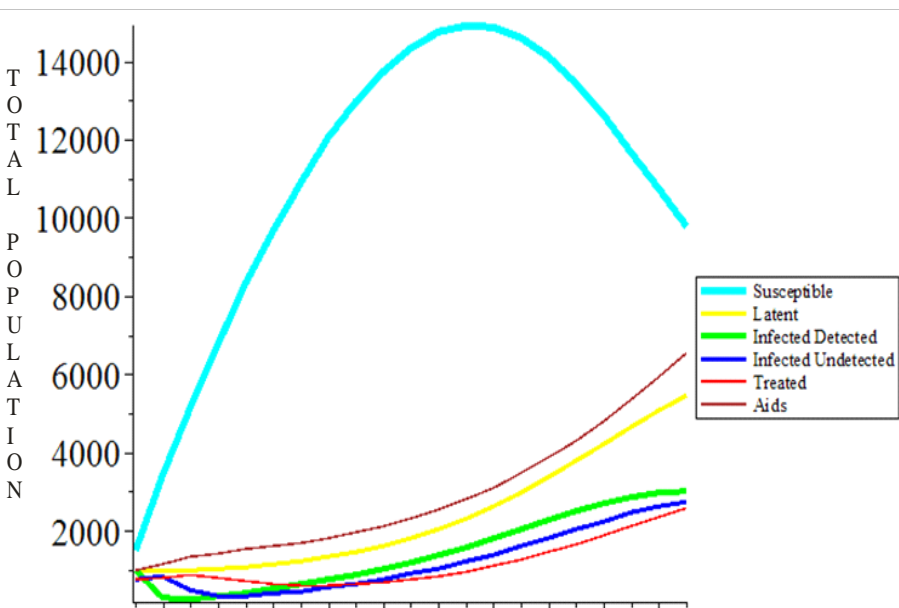

$\begin{array}{lllllllllll}2 & 4 & 6 & 8 & 10 & 12 & 14 & 16 & 18 & 20\end{array}$

Figure 7: Plots of Total Population against time $t$ for various value of $\gamma_{(0.6)}$

$\varepsilon=0.7, \eta_{U}=0.001, \eta_{D}=0.001, \eta_{T}=0.001, \tau=0.30619, \omega=0.2$, $\kappa=0.2522, \pi=2000, \delta=0.3, \rho=0.12, \nu=0.12, \mu=0.02$

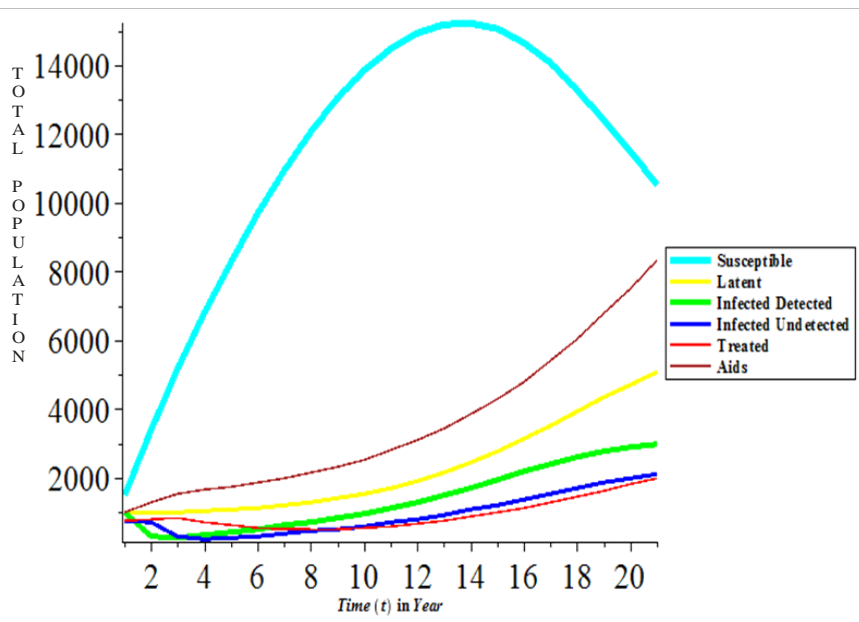

Figure 8: Plots of Total Population time $\mathbf{t}$ for various value of $\kappa_{(0.2522)}$

$\varepsilon=0.7, \eta_{U}=0.001, \eta_{D}=0.001, \eta_{T}=0.001, \tau=0.30619, \omega=0.2$, $\pi=2000, \delta=0.3, \gamma=0.2, \rho=0.12, v=0.12, \mu=0.02$

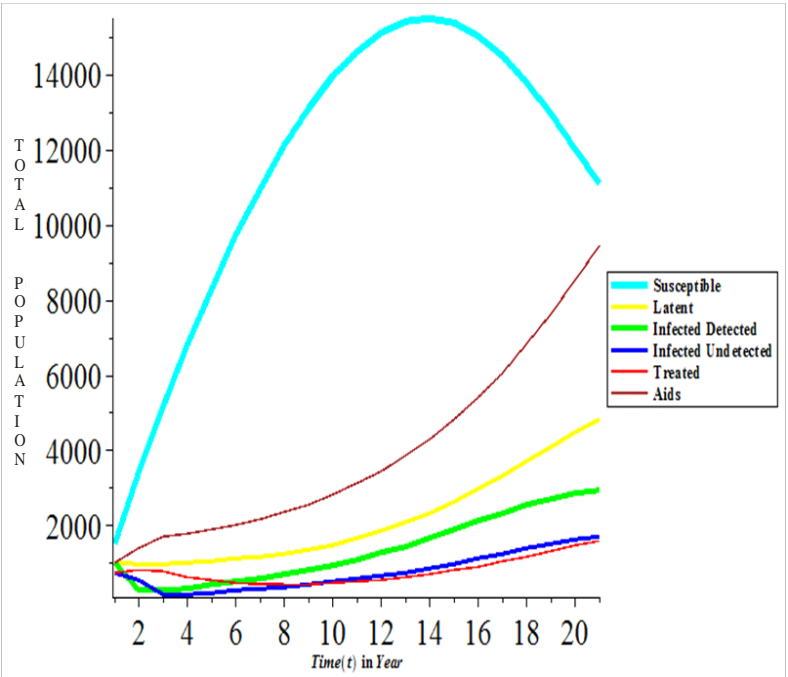

Figure 9: Plots of Total Population against time $\mathbf{t}$ for various value of $\kappa_{(0.6552)}$

$\varepsilon=0.7, \eta_{U}=0.001, \eta_{D}=0.001, \eta_{T}=0.001, \tau=0.30619, \omega=0.2$,

$\pi=2000, \delta=0.3, \gamma=0.2, \rho=0.12, v=0.12, \mu=0.02$

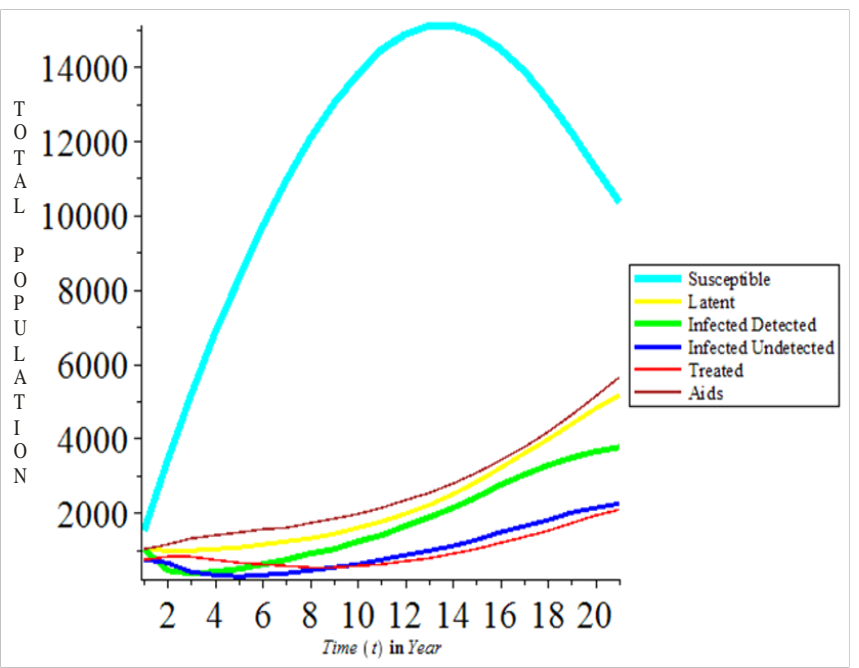

Figure 10: Plots of Total Population against time $t$ for various value of $\gamma(0.4)$

$\varepsilon=0.7, \eta_{U}=0.001, \eta_{D}=0.001, \eta_{T}=0.001, \tau=0.30619, \omega=0.2$,

$\kappa=0.2522, \pi=2000, \delta=0.3, \rho=0.12, v=0.12, \mu=0.02$

\section{DISCUSSION OF RESULTS}

It is observed from the table 3 that when the parameters with positive sensitivity values increases while the remaining parameter remains constant, the value of basic reproduction number increases, and this increase the endemicity of the disease in the population. The increment in basic reproduction number makes it more difficult to have disease free environment. Parameters with negative sensitivity values have 
less effect in the dynamical spread of HIV/AIDS in the population.

Medical practitioners need to work on positive indices parameters most especially parameters that have pronounced effect on basic reproduction $\left(R_{0}\right)$ i.e. transmission rates $\beta$ in order to have stable free disease environment.

Figure 1, 2 and 3 show that effective contact rate $\beta$ has pronounced effect on susceptible, latent and infected undetected individuals. It reduces the population of susceptible individuals and increase the number of latent, Aids, infected detected.

Figure 4 and 5 shows that the higher the detection $\gamma$ of infected undetected individuals, the lower the population of infected undetected and the higher the infected detected individuals. This suggests that, detection of infected individuals plays a vital role in control of HIV disease.

Conclusively, the most sensitive parameter is contact rate ( $\boldsymbol{\beta}$ ) which play a prominent role in the spread of HIV, it was recommended that to reduce the spread of the HIV the detection rate should be given the priority.

\section{REFERENCES}

[1] Castillo-Chavez C., Cooke K., Huang W. \& Levin S.A. (1989). The role of long incubation periods in the dynamics of HIV/AIDS. Part 2: multiple group models. Mathematical and statistical approaches to AIDS epidemiology. Lecture notes in Biomathematics. Springer verlag. 83:200-217.

[2] Eze, J.I. (2009).Model HIV/AIDS in Nigeria. Phd. Dissertation, University of Glasgow.

[3] Hethcote, H. (2000). The mathematics of infectious diseases. SIAM Review, 42(4), 599-653.

[4] Malioyoni, M., Mwamtobe, P.M.M., Hove-Musekwa,S. D.\&Tchuenche, J.M.(2012). Modeling the role of diagnosis, treatment, and health education on multi drug resistance tuberculosis dynamics. ISRN Biomathematics, Article ID459829,120.doi:10.5402/2012/459829.

[5] McCluskey, C.C. (2003). A model of HIV/AIDS with staged progression and amelioration.

[6] Trimpathi, A., Naresh, R. \& Sharma, D. (2007). Model the effect of screening unaware infective on the spread of HIV infection. Science Direct Applied Mathematics and Computation, 184, 1053-1068.

[7] UNAIDS/WHO (2005), Question and Answers III Selected issues; Prevention and Care, http://www.unaids.org/. Retrieved on 10th October, 2010

[8] UNAIDS (2008).UNAIDS: Report on the Global AIDS Epidemic.

www.unaids.org/en/KnowledgeCentre/HIVData/Epid emiology/epidemiologySlidesAuto.asp
[9] UNAIDS (2013). Global Report: UNAIDS report on the global AIDS epidemic. http://www.unaids.org/en/media/unaids/contest assets/documents/epidemiology /gr2013/unaids-global-report-2013-en.pdf

12013

